\title{
e-Phaïstos
}

e-Phaïstos Revue d'histoire des techniques / Journal of the history of technology

VI-2 2017 | 2018

La diversité du patrimoine technique africain

\section{La technique de pêche au Zemi : un savoir-faire en voie de disparition dans les abords du lac Tchad}

The Zemi Fishing Techniques: a Disappearing Know-How along the Banks of the Chad Lake

\section{Ousman MAHAMAT ABBA}

\section{OpenEdition}

\section{Journals}

Édition électronique

URL : http://journals.openedition.org/ephaistos/3407

DOI : 10.4000/ephaistos.3407

ISSN : 2552-0741

Éditeur

IHMC - Institut d'histoire moderne et contemporaine (UMR 8066)

Référence électronique

Ousman MAHAMAT ABBA, «La technique de pêche au Zemi : un savoir-faire en voie de disparition dans les abords du lac Tchad », e-Phaïstos [En ligne], VI-2 2017 | 2018, mis en ligne le 16 novembre 2018, consulté le 01 mai 2019. URL : http://journals.openedition.org/ephaistos/3407 ; DOI : 10.4000/ ephaistos.3407

Ce document a été généré automatiquement le 1 mai 2019.

Tous droits réservés 


\section{La technique de pêche au Zemi : un savoir-faire en voie de disparition dans les abords du lac Tchad}

The Zemi Fishing Techniques: a Disappearing Know-How along the Banks of the Chad Lake

Ousman MAHAMAT ABBA

\section{Introduction}

1 La production économique de chaque groupe humain dépend de son environnement naturel. Les échanges intercommunautaires, l'évolution technologique et les changements climatiques sont des facteurs qui ont incité les Kotoko à innover pour s'adapter aux exigences de leur environnement. L'évolution technique des pirogues leur a permis d'améliorer la production halieutique. Puis, des techniques de conservation plus appropriées développées dans cette région ont favorisé l'exportation du poisson. À propos de la maîtrise des moyens de locomotion sur les cours d'eaux de la plaine tchadienne, le Commandant Lenfant affirmait que : « Les Kotoko sont les seuls indigènes exerçant la profession de piroguiers dans toute la région jusqu'au Lac Tchad. Ils montent de grandes embarcations faites de troncs d'arbres taillés et cousues à la ficelle et dont l'avant est plus large que l'arrière $»^{1}$. Seignobos et Magnant se sont intéressés aux rapports entre ces peuples et leur environnement immédiat ; le premier indique que « les Kotoko peuplent, au Tchad, le Bas-Chari et le Bas Logone et leur économie est celle des gens de fleuve $»^{2}$.

2 L'expression "gens de fleuve " renvoie aux peuples de pêcheurs ou, mieux, à ceux qui opèrent régulièrement sur les cours d'eau; le second auteur fournit des précisions sur la valorisation des services bateliers : « Toute traversée des fleuves en pirogue se fait contre 
le paiement d'un prix qui inclut, outre le travail du piroguier, les redevances du maitre du fleuve ${ }^{3} . .$. ». Hans Dominik décrit en 1908, l'activité de pêche dans la localité de Kousseri : «Toute la ville sentait le poisson et partout, la plage était couverte d'écailles et de déchets de poissons. Dans les nombreuses maisons, de véritables fumeries étaient installées et l'on voyait rarement les makarhs, qui sont de stature élevée et vêtus de vêtements bleus et noirs, autrement que munis d'une rame, de gaffes, de filets ou instruments de pêche ${ }^{4}$. Ces propos traduisent concrètement l'impact de la pêche. L'abondante production halieutique résultait de l'emploi du Zemi, un administrateur colonial français en décrit la procédure : «Une grande barque de pêche avec un immense filet en proue se poste le long de la rive, le filet immergé. Une petite pirogue vient à sa rencontre en remontant le courant. À une trentaine de mètres, un enfant placé en proue de la petite pirogue, frappe le rebord de l'embarquement avec deux courts bâtons ${ }^{5} »$. Les différents témoignages relatifs aux savoir-faire en matière de pêche contribuent aujourd'hui à matérialiser l'histoire économique et sociale de l'aire culturelle kotoko. Ce travail de reconstitution s'intéresse au Wam-zemi, un instrument de pêche qui est une spécificité des Kotoko. L'équipement de pêche est composé d'une grande pirogue (wam), moyen de locomotion, et d'un filet triangulaire (zemi) qui constitue l'instrument de capture du poisson. Cet engin, destiné à la conduite d'une campagne de pêche collective, se présente aujourd'hui aux yeux des populations locales comme l'œuvre la plus achevée du génie kotoko. Plusieurs modèles miniaturisés sont exposés dans les musées ethnographiques de cette aire culturelle et les conservateurs des musées les présentent aux visiteurs avec beaucoup d'enthousiasme.

Figure 1. Carte de localisation du pays kotoko dans le bassin tchadien

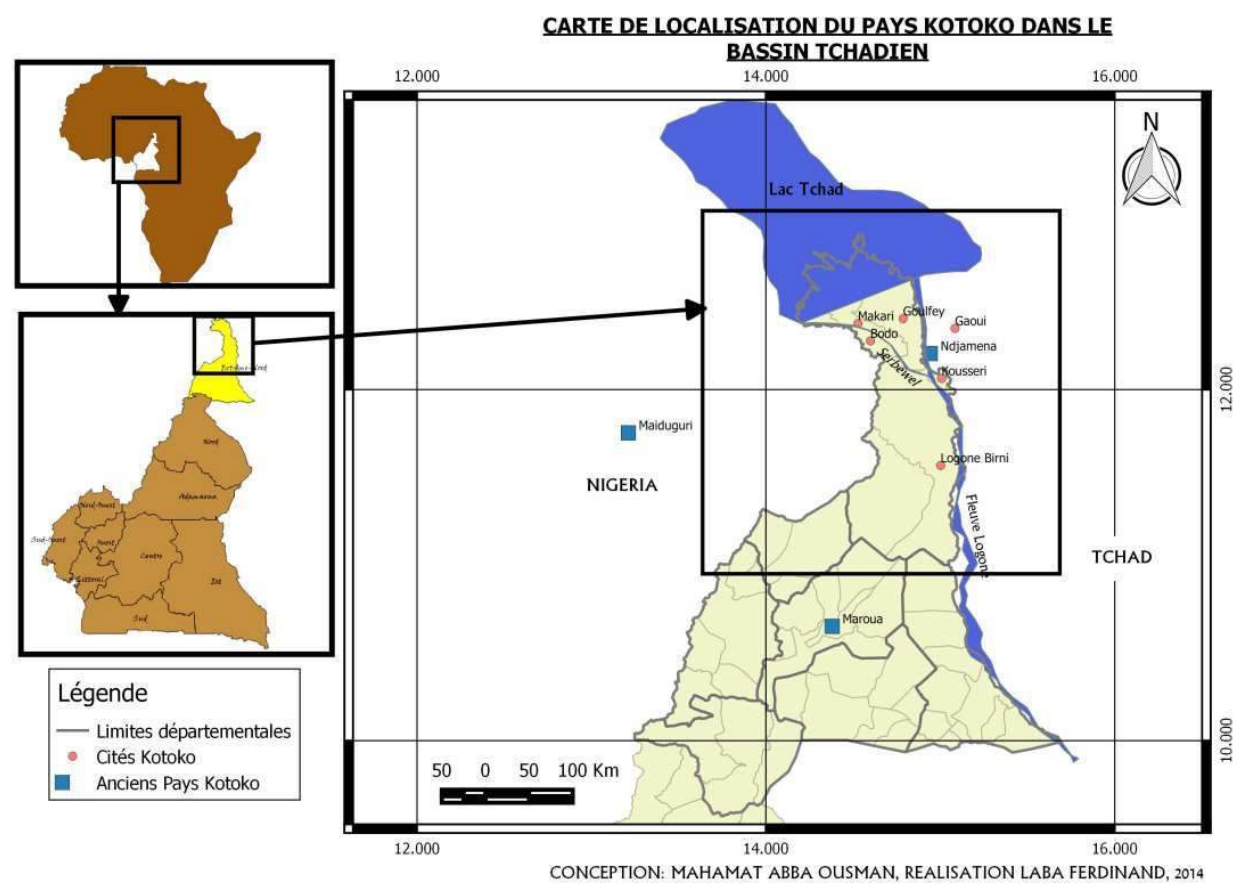

Conception : Mahamat Abba Ousman, réalisation : Laba Ferdinand, 2014

Dès lors, quelle est donc la spécificité de cet outil de pêche et quel fut son impact dans la vie de ces populations? Pour mener à bien cette réflexion, nous nous proposons de faire un aperçu de l'activité de pêche dans cette aire culturelle et de faire une présentation 
physique du Zemi. Puis, cette réflexion nous conduira dans les méandres de la confection de cet engin et son incidence sur la vie socio-économique. Les raisons de sa disparition sur les cours d'eaux feront l'objet de la dernière partie de ce travail.

\section{L'activité de pêche en pays kotoko}

4 La pêche s'est imposée comme la principale activité économique dans les abords sud du lac Tchad. C'est pour cette raison que l'on dénombre des rites et plusieurs imbrications historiques de cette activité dans cette partie de l'Afrique ${ }^{6}$. Le développement de cette activité est certainement lié à la proximité et à la permanence des cours d'eau durant toute l'année. Les rivières les plus exploitées au cœur du pays kotoko sont le Logone, le Chari, l'Elbeit, le Taftaf et le Serbewel, lesquelles constituent les principaux affluents du Lac Tchad. L'image que les voyageurs gardent communément des Kotoko est liée à la pêche. À ce sujet, des chercheurs précisent: "La population riveraine est formée essentiellement d'islamisés. La plus importante et la seule dont l'occupation soit ancienne, est la race kotoko qui a acquis une certaine célébrité par le folklore et ses coutumes de pêche [...]. Les Kotoko emploient de multiples engins hautement spécialisés ${ }^{7}$ ». Jean Paul Lebœuf dresse le tableau d'une flottille en face à la capitale tchadienne ${ }^{8}$ : "Les tours de Zemi, lorsqu'elles faisaient leur campagne de pêche au confluent du Logone et Chari, en face même de Ndjamena, semblaient des libellules légères posées sur le fleuve comme pour narguer les hippopotames qui poussaient hors de l'eau, leurs mufles mugissants. Les filets relevés scintillaient, les cormorans disputaient aux pêcheurs les poissons échappés, tandis que les pélicans voguaient dans le calme. Il a suffi de quelques hors-bords plaisanciers et douaniers pour chasser tout cela ${ }^{9}$ "

5 En somme, il est à noter que le milieu naturel a prédisposé les Kotoko à choisir la pêche comme principale activité. Il semble aussi qu'une réelle volonté ait conduit ce groupe ethnique à développer ce mode de prélèvement halieutique, car ils se sont particulièrement illustrés dans le déploiement de multiples techniques propres à l'industrie de la pêche à l'instar du Wam-zemi, objet de cette étude.

\section{Le Wam-zemi : Un instrument de pêche exceptionnel dans la plaine tchadienne}

Il est constitué de deux parties distinctes. Il s'agit du Wam qui est la pirogue dans laquelle les pêcheurs exercent leur activité, et du filet triangulaire placé à l'avant de la pirogue qu'on appelle Zemi. La conception générale est très ingénieuse et la construction nécessite une attention particulière car une erreur, aussi minime soit-elle, peut entraîner un recommencement des travaux de peur que les pêcheurs ne soient en difficulté pendant la campagne de pêche. La dissymétrie de la pirogue est atypique puisque l'avant, large et camus, est à peine relevé alors que l'arrière est élancé et effilé. Un système pivotant permet de plonger le Zemi dans l'eau grâce à un madrier robuste qui sert de bras de levier. Il s'insère dans la partie pivotante à l'angle droit par rapport aux antennes. Il se couche au fond de la pirogue quand le filet est relevé et se dresse comme un mât incliné quand le filet est orienté vers le fond; l'avant de la pirogue supporte le Zemi (voir fig.2) ${ }^{10}$. 
Figure 2: Une représentation du Wam-zemi

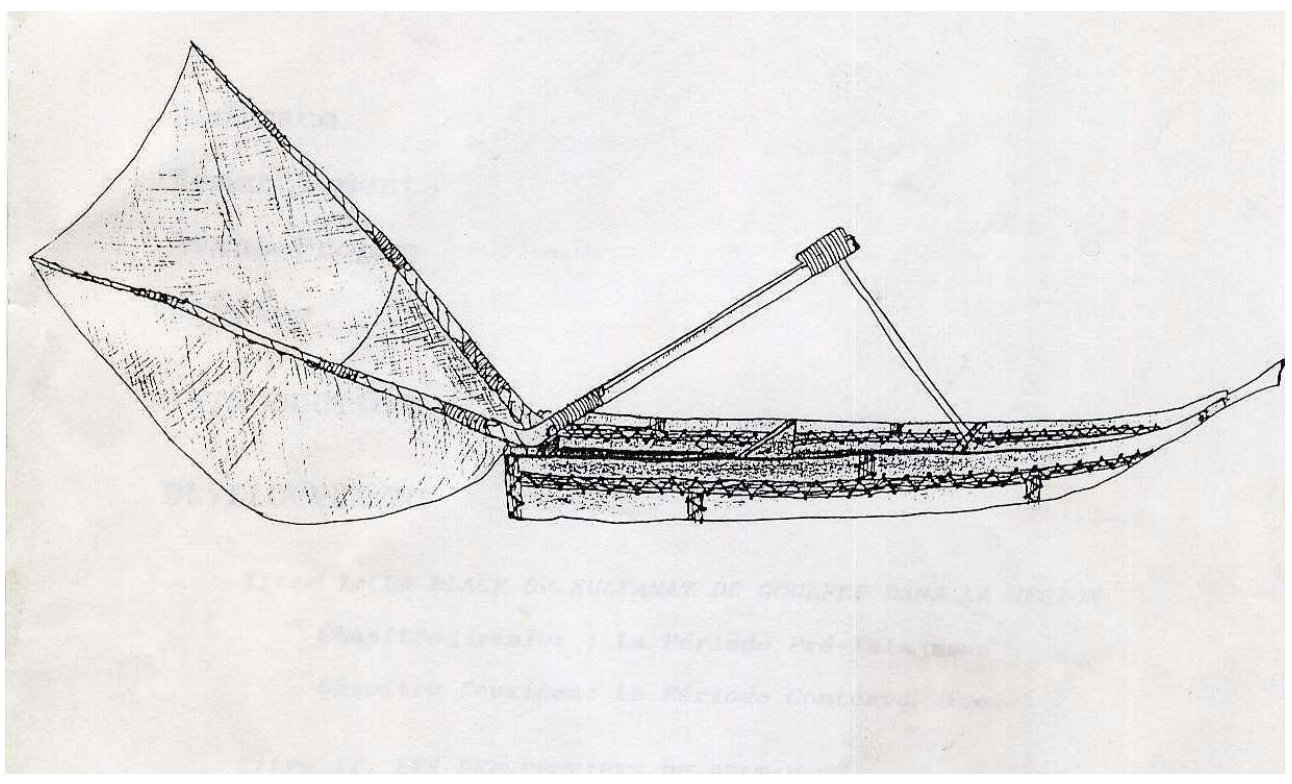

(c) Amicale « Goulfey avenir »

7 La fabrication du Wam se fait sur une période de deux semaines lorsque la main-d'œuvre est constituée de huit personnes au moins. Après l'obtention de l'autorisation des autorités traditionnelles, les artisans disposent d'une semaine entière pour collecter bois et matériaux appropriés dans le périmètre du sultanat. Pour l'essentiel, les essences recherchées se caractérisent par leur grande taille et leur aptitude à flotter aisément sur les eaux (Voir tableau 1) ${ }^{11}$.

Tableau 1. Les essences utilisées pour la fabrication du Wam

\begin{tabular}{|l|l|l|}
\hline Noms scientifiques & Familles & Nom local \\
\hline Mitragyna inermis & Rosaceae & Herr \\
\hline Acacia senegal & Mimosaceae & Ngar \\
\hline Acacia ahreubergiana & Mimosaceae & Mendade \\
\hline Acacia albida & Mimosaceae & Alfe \\
\hline
\end{tabular}

Ensuite, les planches issues de ces arbres sont perforées, puis reliées entre elles par des lianes associées au coton et à des brindilles (voir fig.3). 


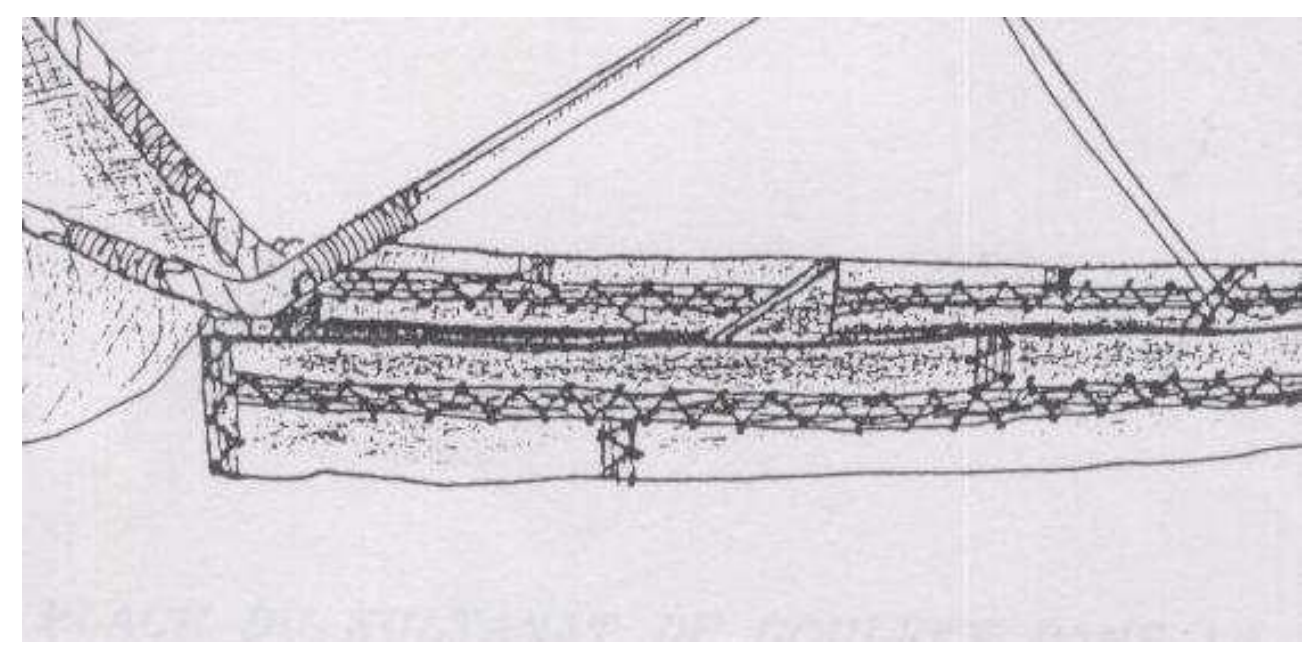

La figure montre les différentes parties reliées par les lianes

(c) Amicale « Goulfey avenir »

Puis, un lieu de travail approprié est choisi, non loin d'un cours d'eau, pour installer l'atelier de fabrication de la pirogue. La main-d'œuvre requise pour atteindre l'objectif est constituée de deux sculpteurs ou tailleurs de bois, d'artisans relieurs et de manœuvres, ces derniers ouvriers sont affectés aux travaux ne requérant pas un savoir-faire particulier. Ils appliquent les instructions des sculpteurs pendant la semaine que dure au minimum le chantier. Les pêcheurs s'accordent sur le fait que la possession d'une pirogue n'est pas à la portée d'un seul particulier dans la mesure où son acquisition génère des dépenses très importantes consécutives au concours de techniciens, d'ouvriers et d'intervenants de proximité originaires d'un même quartier ou d'un petit village qu'il faut solliciter.

Le Zemi se compose d'un grand filet monté sur deux longues antennes qui atteignent 10 à 12 mètres de longueur. Les bases de chaque antenne sont jointes et sont fixées à l'extrémité de la partie avant du Wam où elles reposent; elles s'écartent vers le haut et forment un $\mathrm{V}$ dans lequel s'inscrit un filet de forme triangulaire. La pirogue mesure environ huit mètres de long. Contrairement aux pirogues ordinaires, la partie avant du Wam présente un tableau d'une largeur de 1,5 mètre environ, alors que la partie arrière est semblable à une queue de poisson dont l'extrémité se réduit progressivement, formant une pointe de 0,50 mètre de large ${ }^{12}$. L'association des deux éléments représentés par la pirogue et l'appareil de pêche composent un ensemble appelé Wam-zemi.

Hans Dominik formule la description suivante: «Les grandes barques ancrées en bon nombre sur la rive argileuse rappelaient également les dahabiens ${ }^{13}$. Elles avaient jusqu'à huit mètres de long et deux mètres de large et étaient constitués de madriers, bâties, carrées à l'arrière et dotées d'un bec pointu élevé à l'avant ${ }^{14} »$. C'est ainsi que chaque Wam-zemi est assisté d'une pirogue monoxyle ordinaire où deux pêcheurs émettent un bruit à l'aide d'un dispositif en bois installé à l'intérieur de la petite pirogue dans le but d'effrayer les poissons en les chassant vers le Zemi. Le bruitage est produit à l'aide de deux petits morceaux de bois entrechoqués sur un morceau plus grand placé à l'intérieur de la pirogue. Le son émis par ce dispositif est comparable à l'onomatopée « ko-to-ko-to-ko » 
qui serait à l'origine de la dénomination Kotoko attribuée ce peuple de pêcheurs ${ }^{15}$ connu davantage sous l'appellation Mantagué, Lagwané ou Mser ${ }^{16 .}$

Le système de pêche Wam-zemi intervient comme un symbole de l'identité culturelle des Kotoko dans la mesure où sa fabrication recourt à des matériaux locaux et matérialise un savoir-faire vernaculaire original. Jean-Claude Zeltner qualifie cette activité de prélèvement de "pêche industrielle", car elle mobilise plusieurs personnes et la production est assez élevée ${ }^{17}$. Si la mise en œuvre de cette pratique s'observe toute l'année, elle est plus productive durant la période allant du mois décembre au mois de mai ${ }^{18}$. La reproduction nous montre le déploiement d'un zemi servi par un équipage de quatre pêcheurs sur un cours d'eau ${ }^{19}$ (photo $\left.n^{\circ} 1\right)$.

Photo $n^{\circ} 1$ : Une pirogue wam équipée d'antennes ou zemi

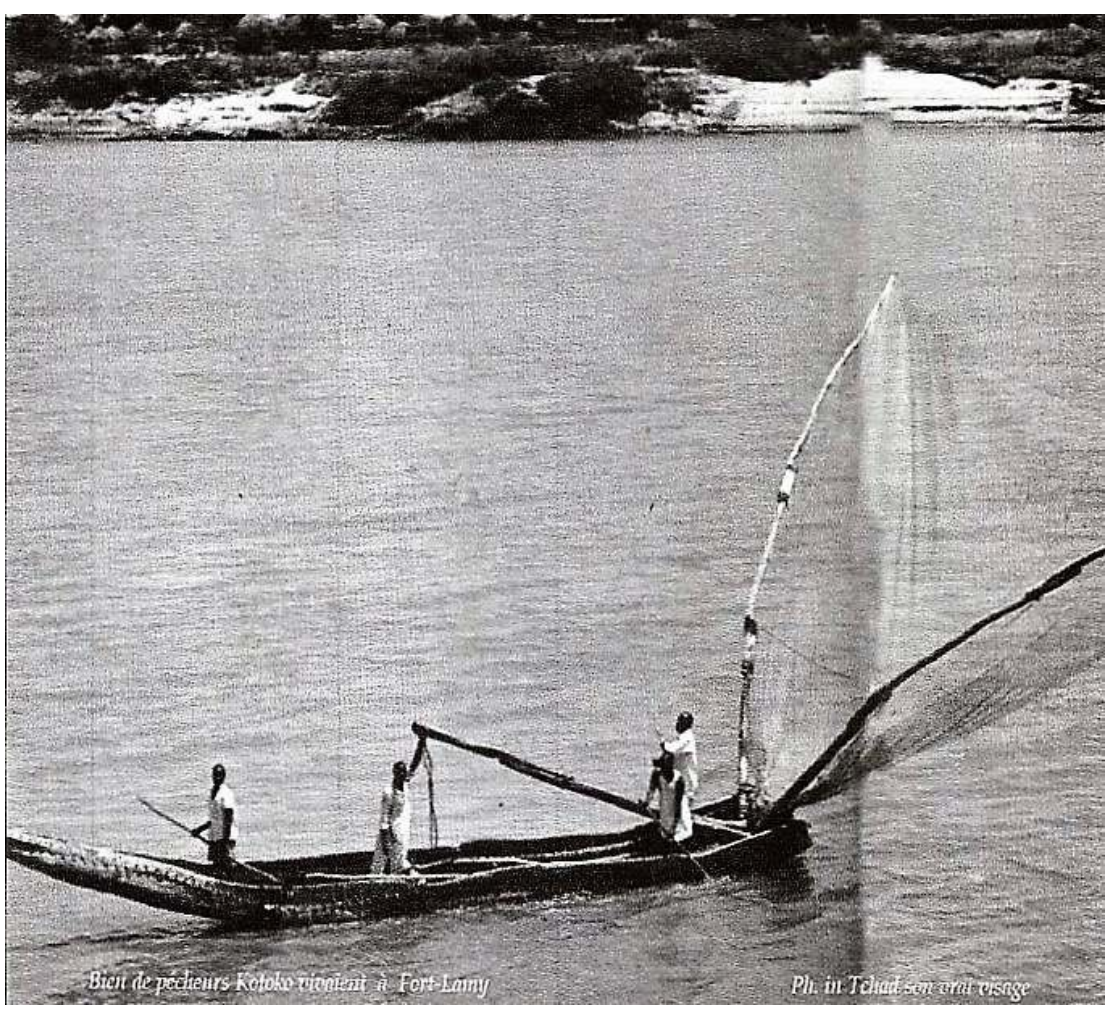

(c) Alhadji Alifa Mey Eli

\section{La pêche au Zemi, une activité économique majeure dans la plaine tchadienne}

Cette technique de pêche, très rentable du point de vue économique, a gagné progressivement l'ensemble du pays kotoko: «il est intéressant de constater l'augmentation du nombre de ces grandes embarcations depuis les recensements du professeur Monod (en 1929: 68 Zemis; en $1955: 169$ Zemis $^{20}$ ) . Cette augmentation fut liée à la forte demande de poisson émise par les consommateurs du Nigeria. Une campagne de pêche au Zemi mobilisait la plus grande partie de la population d'un village : 10 à 12 hommes formaient l'équipage. Au campement de pêche, une dizaine de personnes étaient chargées du traitement des poissons frais, de la surveillance et de la commercialisation du produit transformé. La capacité de charge de la pirogue est forte: les dimensions 
moyennes sont estimées à $1,50 \mathrm{~m}$ de largeur extrême, à $0,65 \mathrm{~m}$ de creux intérieur et à 12 $\mathrm{m}$ de long ${ }^{21}$ Cependant, les Wam-zemi des années mille neuf cent soixante-dix étaient nettement plus petits et un équipage de six hommes suffisait pour les manœuvrer. Chaque équipe de pêche était constituée d'une dizaine de pêcheurs répartis entre le Wamzemi et la petite pirogue accompagnatrice. Postes et responsabilités étaient définis de manière précise. Le chef de bord se tenait à l'arrière de la grande pirogue et avait la lourde tâche de diriger le Wam-zemi avec sa pagaie. Deux personnes situées sur chaque bord, au milieu de l'embarcation assistaient le chef de bord dans la conduite de la pirogue. Deux autres personnes se livraient à la manœuvre du filet à l'aide du bras de levier, soit pour vider les poissons capturés dans la pirogue en le levant, soit pour le plonger dans l'eau en le baissant. Les cinq autres personnes récupéraient les poissons, les triaient et les vidaient immédiatement afin d'éviter que le produit de la pêche ne s'abîme au cours des longues heures que comptait une séance de capture. Ce travail à la chaîne exigeait une rigueur et un engagement total des assistants. La pêche au Zemi pouvait être pratiquée de manière continue toute la journée ou bien la nuit entière selon le déroulement de la campagne de pêche ${ }^{22}$.

Des dispositions étaient prises pour que les pêcheurs puissent se faire à manger pendant qu'ils étaient en pleine activité. Deux foyers en terre cuite étaient installés dans la pirogue, à l'usage d'une femme choisie par le chef de bord: la sœur, l'épouse ou la bellesœur du chef d'équipage. Elle prenait toutes les dispositions nécessaires à l'alimentation des pêcheurs ${ }^{23}$. Dans la pratique, elle contractait un marché avec les pêcheurs pour la fourniture des ingrédients nécessaires à la préparation des repas, notamment la farine pour le couscous, le sel, le piment, l'huile de poisson, les oignons, le sucre, la poudre de thé et le bois de cuisson. La cuisinière était payée après la vente du poisson. Elle recevait quotidiennement une quantité de poisson qui lui permettait de renouveler les produits alimentaires. De cette façon, les pêcheurs ne s'arrêtaient que le temps d'un repas préparé sur les foyers amovibles en terre cuite et consommé dans la pirogue. Cette organisation pourrait justifier l'appellation "pêche industrielle » employée par certains chercheurs dans la mesure où elle mobilisait des moyens humains, financiers et techniques de grande envergure.

Du fait qu'il s'agissait d'une organisation collective de la pêche, une gestion spécifique des zones de pêche était observée par les différents groupes de pêcheurs. Dans le sultanat de Goulfey, les autorités traditionnelles avaient subdivisé les berges du Logone en quatre grands secteurs de pêche. Il s'agissait de la zone de pêche de Mougalam, le campement d' Afié, de mouskondo et de molodia ${ }^{24}$. La flottille de pirogues était répartie sur ces quatre Gaya ${ }^{25}$ et une rotation des secteurs de pêche s'effectuait tous les deux jours, pour permettre à tout le monde d'avoir les mêmes chances de profit. En une semaine, tous les pêcheurs parcouraient les quatre secteurs de pêches subdivisés par les notables en charge du contrôle de cette activité. Cette gestion du domaine de pêche était capitale car elle permettait d'éviter les disputes entre pêcheurs, en raison de l'inégalité de la richesse halieutique des différentes zones de pêche. 


\section{La conservation de ressources halieutiques en pays kotoko}

Des techniques impressionnantes en matière de traitement des produits de la pêche étaient développées pour la conservation et l'exportation du poisson dans l'ensemble du bassin tchadien et même dans la sous-région d'Afrique centrale, notamment à Ndjamena, à Bangui, à Yaoundé, à Maiduguri et dans le septentrion camerounais ${ }^{26}$. Il est reconnu que la ville de Maroua était le point de convergence de cette production depuis des décennies. «Maroua, est le principal relais de collecte et de distribution du poisson frais ou transformé (fumé ou séché) dans cette région. Le marché hebdomadaire du lundi est celui qui attire le plus, les commerçants et les consommateurs de poissons qui viennent parfois des confins de la province pour s'approvisionner en poisson au lieu-dit Village du Comice $27 \%$

La vulgarisation des techniques de conservation se substituaient à l'absence de possibilité de conservation par le froid. Les populations avaient recours au fumage et au séchage car ils n'avaient la possibilité d'écouler le poisson frais qu'à Ndjamena et dans la ville de Kousseri où la demande était relativement faible et le prix d'achat dérisoire. Ainsi, les épouses et les enfants de pêcheurs tronçonnaient les gros poissons destinés au boucanage. Des fours en pisé étaient construits autour des campements de pêche et derrière les demeures des pêcheurs, tandis que les campements de pêche étaient autour des cités fortifiées. Les grillages en fer étaient usités comme supports sur lesquels étaient déposés les poissons frais destinés au fumage. Puis, grâce au feu du bois, les poissons étaient fumés à longueur de journée sous le contrôle d'un chef de camp ${ }^{28}$. Les poissons fumés appelés localement Banda, étaient destinés à l'exportation. Blanche J. \& alii présentent ainsi les poissons fumés : «Les poissons donnent un produit correct, à goût agréable [...] emballé dans des paniers spéciaux en papyrus vert et exportés vers le Nigeria dont les marchés sont très proches ${ }^{29} »$. Ces emballages traditionnels ont disparu progressivement du circuit de vente pour deux raisons: la première relevait de l'exploitation abusive de cette essence végétale par les pêcheurs exportateurs de poisson fumé. Ils ne donnaient pas aux plantes la possibilité de se reconstituer. Puis, face à l'accroissement de l'exportation du poisson il fallut trouver un moyen de substitution pour pallier la disparition du papyrus. Cette situation contraint les pêcheurs à utiliser des cartons importés du Nigeria à la fin des années mille neuf cent soixante ${ }^{30}$. À la technique de conservation du poisson par fumage s'ajoutait celle du séchage.

La seconde technique de conservation de la production halieutique fut employée pour deux raisons. Certaines espèces de poissons se décomposent très rapidement et comme, dans les campements de pêche, le fumage n'a lieu que le lendemain, les pêcheurs sont contraints de procéder au séchage. La seconde raison est liée à la taille de certaines espèces, qu'il est impossible de placer sur le grillage. Toutefois, même si les pêcheurs disposaient d'un grillage à maille réduite, le fumage de poisson de petites tailles reste pénible $^{31}$. Le fumage est délicat puisqu'il faut suivre le processus régulièrement pour éviter des désagréments. C'est ainsi que la technique du séchage s'est imposée dans cette région.

19 Il s'est développé un métier d'éventreurs de poissons destinés au séchage en masse autour des campements de pêche. Il s'agit en réalité d'une sous-traitance dans cette activité en pays kotoko. Le paiement de cette main-d'œuvre, en grande partie recrutée au 
sein de la famille ou dans quartier, s'effectuait en nature. C'est ainsi que, pour dix poissons éventrés, un poisson de même taille revenait de droit à l'éventreur ${ }^{32}$. Pour le fumage, les pêcheurs permettaient à leurs assistants d'exploiter les têtes de poissons à leur propre compte. Ils recevaient leur indemnité après la vente du poisson. Il est donc possible que le mode de payement de « ces techniciens » tire ces origines du réalisme des pêcheurs au regard des contraintes de temps. Cette technique a marqué plus d'un voyageur à cause de la souplesse avec laquelle le poisson était traité sans qu'aucune arête du poisson ne soit endommagée.

Figure 4 : technique de séchage des gros poissons

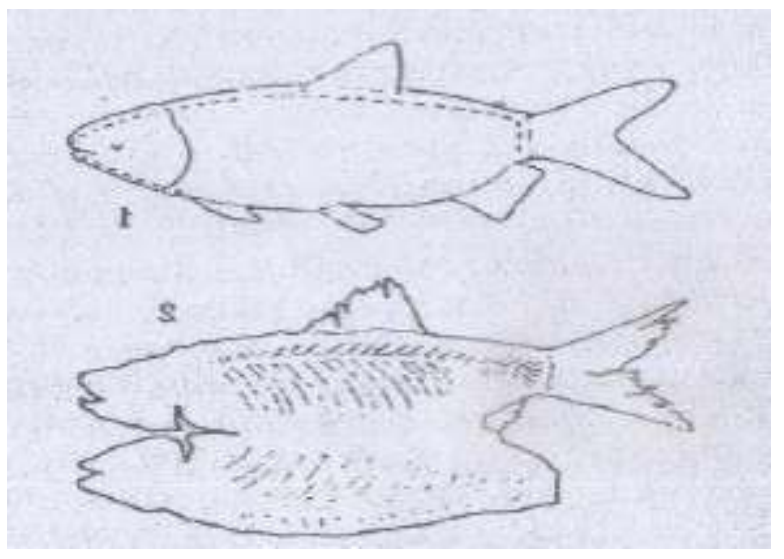

(c) Creac'h Paul 1993

Tchad, 1937-193933 témoigne de l'originalité et de l'unicité de cet art en matière de conservation des produits de la pêche. Appliquée exclusivement aux gros poissons, cette technique permet aux femmes de recueillir l'huile, qui dérive conjointement de la décomposition du poisson et de l'action du soleil. Cette huile est très prisée dans cette région. Elle intervient dans la cuisson des aliments. Les populations s'en servent dans plusieurs domaines. Elle est considérée comme un aphrodisiaque au même titre que l'huile de morue. Elle est d'une utilité alimentaire évidente et ses vertus thérapeutiques sont reconnues localement de manière empirique. Les Kotoko assaisonnent tous les repas avec cette huile malgré son goût âcre ${ }^{34}$. Paul Creac'h indique son utilité au sein de cette communauté : «En saison de pluie, quand les enfants ne sont pas forts, ils boivent l'huile de poisson. C'est un aliment qui procure de la chaleur et qui donne des forces $»^{35}$. Ce produit a aussi des propriétés purgatives lorsqu'il est mélangé au natron et absorbé rapidement en grande quantité. Les pasteurs arabes versent aussi cette huile dans les naseaux des animaux qui toussent au sein du cheptel ${ }^{36}$.

La pêche au Zemi a donc occupé une place importante dans les activités économiques. Ainsi, au lendemain des indépendances en 1960, la partie camerounaise de l'aire culturelle kotoko intégra la circonscription administrative du Logone et de Chari où, en 1963, le préfet décida de regrouper les pêcheurs au sein d'une coopérative ${ }^{37}$ dénommée «SOCOPELI » (Société Coopérative de Pêche du Logone et Chari). Celle-ci a vu le jour le 14 novembre à Kousseri. Les autorités traditionnelles kotoko étaient fortement impliquées dans la mise en place de cette structure. C'est ainsi que Ali Mahamat, sultan de Goulfey a été membre de la coopérative tandis que le sultan de Kousseri, Mahamat Moussa, faisait partie des six administrateurs de la structure. Ali Mahamat a d'ailleurs présidé aux 
destinées de la SOCOPELI de 1960 à 1978. Ce poste lui a permis de travailler en étroite collaboration avec la chambre d'agriculture dont il est membre depuis le 26 novembre 1963. Il a occupé dès lors des postes à responsabilité importants ${ }^{38}$. Il fut nommé Viceprésident suppléant, président par intérim de 1985 à 1987 et président de la Section Élevage de 1987 à nos jours ${ }^{39}$. Ainsi, l'activité de la pêche a permis à un fils de l'aire culturelle kotoko d'intégrer la plus haute structure en charge des questions de pêche au Cameroun. Aujourd'hui, les chantiers de construction de wam-zemi, et les intenses activités de pêche ont disparu du paysage culturel et économique de l'aire culturelle kotoko avec leurs savoir-faire. Les conservateurs des musées ethnographiques ont donc commandé des Zemis miniaturisés qui sont exposés dans six structures muséales pour informer les touristes sur le passé de cette région. Se dégage aussi le désir d'indiquer, à la jeune génération du pays kotoko, que ces engins ont marqué la conscience collective de leur communauté; les visiteurs ne manquent pas de témoigner leurs émotions et surprises, par leurs écrits. D'où la nécessité de s'interroger sur la disparition de cet outil de pêche dans les milieux piscicoles des abords sud du lac Tchad.

\section{La disparition du Zemi sur les eaux du Logone}

De nos jours, on n'observe plus ces pirogues sur les cours d'eau de la plaine tchadienne. Plusieurs explications nous sont fournies par les différents acteurs en présence. Les pêcheurs indiquent que toutes ces pirogues ont été démontées et stockées vers les années 1972 parce que l'on ne pouvait plus recruter d'équipage. Une partie de la population estime que la disparition de cette technique de pêche est tributaire de la raréfaction du poisson dans les rivières des abords du lac Tchad. Et Jean-Paul Lebœuf de dire que « Les coopératives de pêche ont tué les Zemi traditionnels, mais par un retour imprévu, les circuits commerciaux traditionnels ont asphyxié et tué les coopératives ${ }^{40} »$. Ce bouleversement serait lié au fait que les populations n'étaient pas fortement impliquées dans la mise en place et la gestion cette coopérative. Un examen approfondi de ce constat ne peut pas exclure la thèse du réchauffement climatique et ses effets comme l'irrégularité des pluies, la déforestation et l'avancée du désert. Notamment, l'absence des pluies a pour conséquence la réduction des zones de cantonnement pour la reproduction des poissons.

Nous pensons aussi qu'une étude plus poussée doit être faite pour faire un inventaire des espèces halieutiques disponibles dans les eaux du Logone et du Chari. Ce travail d'expertise pourrait donner des informations sur l'inventaire quantitatif et qualitatif des ressources halieutiques disponibles afin de se prononcer sur les raisons réelles de l'absence des poissons dans les eaux de l'aire culturelle kotoko. Ainsi, l'on pourrait s'interroger sur les techniques de capture, les espaces de reproduction des poissons et le réchauffement climatique caractérisé par l'absence des inondations telles observées au début des années 1960 et 1970 . Ce travail pourrait aussi nous permettre de nous prononcer avec certitude sur la disparition des Zemi bien que nécessitant des moyens colossaux pour son fonctionnement.

Entre 1972 et 1980, ces grandes pirogues furent réservées au transport de personnes et de biens. Les populations s'en servaient pour les voyages de longues distances, pour assister aux mariages et autres manifestations culturelles dans les principautés voisines ${ }^{41}$. Pour être plus pratiques et efficaces pour des tels voyages, les pagayeurs réalisaient des abris, d'un mètre de hauteur, qui étaient recouverts de nattes décorées, afin de satisfaire au 
confort et à la commodité des passagers. Ce mode de déplacement a disparu à partir de 1982, année au cours de laquelle plusieurs cités kotoko disposaient d'un grand nombre de véhicules. Des pirogues plus réduites sont toujours utilisées pour le transport de courte distance ou pour une pêche individuelle. La taille de celles-ci varie entre 3 à $4 \mathrm{~m}$ de long et une largeur qui n'excède pas 1 mètre. Elles sont fabriquées généralement à partir d'un tronc d'arbre ${ }^{42}$. Elles permettent la traversée de la rivière Chari par les commerçants. L'usage du Zemi est resté gravé dans la mémoire de ceux qui l'ont vu disparaitre, une nostalgie pour les acteurs de l'histoire économique et sociale de l'aire culturelle kotoko aujourd'hui reconvertie en zone rizicole. Pour la jeunesse kotoko soucieuse de ce patrimoine culturel en péril, le désir de revoir ces engins sur les eaux du Logone est immense.

\section{Conclusion}

En somme, la pêche au Zemi se présente aujourd'hui comme un élément majeur du patrimoine culturel kotoko au regard de son importance dans la vie socio-économique de cette région. Cet instrument de pêche a marqué de nombreux voyageurs qui ont séjourné sur les bords du Logone au début des années 1970, période au cours de laquelle les derniers Zemi opéraient encore sur ses eaux. Son originalité réside dans le choix des matériaux de fabrication, de l'application des savoir-faire locaux et de la capacité de l'engin à produire et à contenir les ressources halieutiques durant une campagne de pêche. Il constitue un élément palpable de l'histoire des techniques dans les bords sud du lac Tchad, et mérite une attention particulière des chercheurs au regard des différents secteurs d'activités des populations de cette région. Pendant plusieurs décennies, le Zemi était au cœur des activités socio-économiques à travers l'exportation des ressources halieutiques dans la sous-région. Puis, les changements climatiques avec ses corollaires tels que l'irrégularité des pluies et la dégradation de l'environnement ont eu un impact réel sur la faune aquatique. La conséquence majeure est l'absence de cet instrument de pêche sur les cours d'eau. Aussi, faut-il que l'exposition des modèles miniaturisés du Zemi dans les musées ethnographiques kotoko témoigne de l'intérêt que ce groupe ethnique accorde à cette technique originale et de la nécessité de pérenniser cet élément du patrimoine culturel local. C'est ainsi que pour attirer l'attention de la jeune génération, les populations de sultanat de Goulfey ont simulé cette activité de pêche au Zemi en février 1998 à l'occasion de la commémoration de cinquante années de règne du sultan Ali Mahamat, sur le trône depuis novembre 1947. Cette démonstration traduit la place de choix qu'occupe l'activité de pêche dans cette aire culturelle. C'est pour cette raison que nous suggérons qu'un festival culturel soit dédié exclusivement à cet outil de pêche et que des scientifiques soient conviés à cet effet pour que des travaux plus exhaustifs soient menés, afin de donner une visibilité à cet aspect de l'histoire des pêcheurs des abords sud $\mathrm{du}$ lac Tchad. Que des pirogues à antennes ou Wam-zemi d'une taille plus importante soient réalisés et déposés dans les différents musées ethnographique kotoko, car les modèles miniaturisés qu'on y retrouve ne représentent pas la portée historique de cet élément du patrimoine culturel. 


\section{BIBLIOGRAPHIE}

ABAKAR Ahmat, Chronographie des 50 ans de règne sultan Ali Mahamat de Goulfey, document inédit, 1998.

BLACHE Jacques, MITON François et STAUCH Alfred, Première contribution à la connaissance de la pêche dans le bassin hydrographique Logone-Chari-Lac Tchad, aspect général des activités de la pêche et de la commercialisation des produits, description des engins de pêche et leur emploi, Paris, O.R.S.T.O.M., 1962.

CREAC'H Paul, Se nourrir au Sahel, l'alimentation au Tchad (1937-1939), Paris, L'Harmattan, Paris, 1993 DOMINIK Hans, De l'Atlantique au lac Tchad. Campagnes et explorations au Cameroun [Vom Atlantik zum Tschadsee. Kriegs- und Forschungsfahrten Vom Atlantik zum Tschadsee], Berlin, 1908.

GUERPILLON M., « Les langues dites « kotoko », Études Camerounaises, 1948, p. 23-24.

LEBCEU Jean-Paul, Étude kotoko : ethnologie, géographie, linguistique, Paris, Mouton, 1976.

LENFANT Eugène, La grande route du Tchad, Paris, Hachette, 1905.

MAGNANT Jean-Pierre, « Gens de la terre et gens de l'eau au Tchad », in JUNGRAITHMAYR Herrmann, BARRETEAU Daniel, et SEIBERT Uwe (éds.), L'homme et l'eau dans le bassin du lac Tchad, Paris, Éditions de l'Orstom, 1997, p. 403-418.

MAHAMAT ABBA Ousman, Le musée de Goulfey: Inventaire des collections et contribution à l'histoire, Mémoire de maîtrise d'Histoire, Université de Ngaoundéré, 2006.

MAHAMAT ABBA Ousman, Le patrimoine culturel kotoko (XX'-XXI siècles) : Source de l'histoire, produit économique et instrument idéologique, thèse de doctorat $\mathrm{Ph} / \mathrm{D}$ en histoire, Université de Ngaoundéré, 2013.

SEIGNOBOS Christian, « Architecture traditionnelle », Tchad et Culture, 112, 1978.

TAIMOU Adjit, Les Kotoko des abords du Lac Tchad : origines, migrations et implantations, mémoire de DIPES II, ENS, Yaoundé, 1994.

ZELTNER Jean-Claude, Les pays du Tchad dans la tourmente, 1880-1902, Paris, L'Harmattan, 1988.

\section{ANNEXES}

Glossaire

Banda : Poisson fumé en arabe

Dahabieh : De l'arabe dahabiyah, bateau du Nil

Gaya : Zone de pêche dans la langue kotoko

Mrena: Nom du génie de l'eau à Logone Birni

Wam : Pirogue

Zemi : Filet triangulaire 


\section{NOTES}

1. LENFANT Eugène, La grande route du Tchad, Paris, Hachette, 1905, p.152.

2. SEIGNOBOS Christian, « Architecture traditionnelle », Tchad et Culture, 112, 1978, p.34.

3. MAGNANT Jean-Pierre, "Gens de la terre et gens de l'eau au Tchad », dans JUNGRAITHMAYR Herrmann, BARRETEAU Daniel, SEIBERT Uwe (éds.), L'homme et l'eau dans le bassin du lac Tchad, Éditions de l'Orstom, Paris, 1997, p.407.

4. DOMINIK Hans, 1908, De l'Atlantique au lac Tchad. Campagnes et explorations au Cameroun [Vom Atlantik zum Tschadsee. Kriegs- und Forschungsfahrten Vom Atlantik zum Tschadsee], Berlin, 1908, p.69.

5. GUERPILLON M., «Les langues dites « kotoko » », Études Camerounaises, 1, 23-24, 1948.

6. De nombreuses activités sont liées à la pêche. Il s'agit de rituels, des fêtes et des techniques de conservation des ressources halieutiques. Les populations continuent à faire des sacrifices aux génies de l'eau en immolant un mouton. La peau, les intestins, la tête et les pattes de l'animal sont offerts aux génies de l'eau : les Mrena. C'est un officiant attitré qui est chargé de l'exécution de cette tâche. C'est à l'issue de ce rituel que la campagne de pêche est ouverte par les autorités traditionnelles.

7. BLACHE Jacques, MITON François et STAUCH Alfred, Première contribution à la connaissance de la pêche dans le bassin hydrographique Logone-Chari-Lac Tchad, aspect général des activités de la pêche et de la commercialisation des produits, description des engins de pêche et leur emploi, Paris, O.R.S.T.O.M., 1962, p.45.

8. La capitale tchadienne était connue sous l'appellation de Fort Lamy en 1900. Elle devint Ndjamena à l'indépendance proclamée le 11 août 1960.

9. LEBCEUF Jean-Paul, Étude kotoko : ethnologie, géographie, linguistique, Paris, Mouton, 1976, p.26.

10. Dessin tiré de la brochure Faire connaissance avec Goulfey éditée par l'amicale «Goulfey avenir » à l'occasion du cinquantième anniversaire de l'intronisation du sultan Ali Mahamat le 28-11-1947, célébré en février 1998.

11. MAHAMAT ABBA Ousman, Le musée de Goulfey: Inventaire des collections et contribution à l'histoire, Mémoire de maîtrise d'Histoire, Université de Ngaoundéré, 2006.

12. Cette description a été élaborée lors d'un entretien collectif avec un groupe d'anciens pêcheurs kotoko, en mai 2010 à Goulfey. Les participants étaient Mahamat Toma, Mey Sara, Moussa Wakelo, Mahamat Iye et Abba Mayo Halima, réunis autour un appareil confectionné spécialement pour faire des démonstrations de cette technique de pêche lors de la célébration des cinquante années de règne du sultan de Goulfey sa majesté Ali Mahamat en février 1998.

13. Soit Dahabieh de l'arabe dahabiyah, bateau du Nil.

14. DOMINIK 1908, op.cit., p. 65.

15. TAIMOU Adji, 1994, Les Kotoko des abords du Lac Tchad: origines, migrations et implantations, mémoire de DIPES II, ENS, Yaoundé, 1994, p.18.

16. MAHAMAT Abba Ousman, 2013, Le patrimoine culturel kotoko (XX'-XXI siècles): Sources de l'histoire, produit économique et instrument idéologique, thèse de doctorat en histoire, Université de Ngaoundéré.

17. ZELTNER Jean-Claude, Les pays du Tchad dans la tourmente, 1880-1902, Paris, L'Harmattan, 1988.

18. Entretien avec Abba Ousman à Goulfey le 2 mai 2008.

19. Archives privées du sultan de Goulfey, photo non datée.

20. BLACHE, MITON, STAUCH 1962, op.cit., p. 21.

21. Entretien avec Moussa Wakelo en mai 2010 à Goulfey.

22. Les pêcheurs utilisent de lampes torches attachées généralement au niveau de leur front. Ils sont aussi aidés parfois par le clair de lune. 
23. Entretien avec Hadja Semba, mai 2010 à Goulfey.

24. Mougalam, Afié, Mouskondo, Molodia : ce sont les noms de villages où les camps de pêche sont installés pendant les périodes propices de capture de poissons.

25. Cette expression signifie « espace de pêche » dans la langue locale.

26. Entretien avec Abba Liman Masse à Goulfey, décembre 2010. Cet ancien grossiste et revendeur faisait le trafic du poisson entre le pays kotoko et la république centrafricaine entre 1962 et 1965. Puis, il s'est tourné vers le marché de la briqueterie en 1966. Au début des années 1980, il s'est reconverti en transporteur, car les produits halieutiques en provenance de Lagdo étaient chers à Yaoundé. En effet, la création du barrage de Lagdo est doublement perçue comme un facteur de régression de l'activité de la pêche autour du lac Tchad. Ces populations estiment que les poissons n'émigrent pas assez à la suite de l'ouverture de cet ouvrage. Le barrage de Lagdo a aussi attiré tous les pêcheurs professionnels de la plaine tchadienne.

27. Ministère des investissements publics et de l'aménagement du territoire, Schéma directeur régional d'aménagement et développement durable du territoire de la province de l'extrême Nord, p.77.

28. Entretien avec Moussa Wakelo, pêcheur reconverti en riziculteur, mai 2010, à Goulfey.

29. BLACHE, MITON, STAUCH, 1962, p.20.

30. Entretien avec un groupe d'anciens pêcheurs kotoko, mai 2010, à Goulfey: il s'agit de Mahamat Toma, Mey Sara Moussa Wakelo, Mahamat Iye et Abba Mayo Halima.

31. Entretien avec Abba Ousman à Goulfey, mai 2010.

32. Entretien avec Moussa Wakalo en mai 2010 à Goulfey.

33. CREAC'H 1993, op.cit.

34. Entretien avec Mahamat Toma à Goulfey, mars 2008.

35. CREAC'H 1993, op.cit., p.244.

36. Entretien avec un groupe d'anciens pêcheurs kotoko, mai 2010 à Goulfey: il s'agit de Mahamat Toma, Mey Sara, Moussa Wakelo, Mahamat Iye et Abba Mayo Halim.

37. Procès-verbal de la réunion du conseil d'administration du 14 Novembre [?] dans la salle de la mairie de Fort-Fourreau, archives privées du sultan Ali Mahamat de Goulfey.

38. ABAKAR Ahmat, Chronographie des 50 ans de règne du sultan Ali Mahamat de Goulfey, document inédit, 1998, p.5.

39. « Nos jours » renvoient à août 2014, le moment où nous faisons des enquêtes sur le terrain.

40. LEBEUF 1976, op.cit., p.26.

41. Entretien collectif avec Mahamat Toma, Mey Sara, Moussa Wakelo, Mahamat Iye Et Abba Mayo Halima, mai 2010 à Goulfey.

42. Entretien avec Mey Eli Sara à Goulfey, mars 2011.

\section{RÉSUMÉS}

Les activités économiques déterminent le mode de vie de groupes humains et constituent dans certains cas le socle même de leur civilisation. Dans le périmètre du Lac Tchad, les Kotoko sont reconnus comme des pêcheurs professionnels au point où l'histoire de ce peuple est intimement liée à cette activité. Ces derniers ont développé, au cours de leur histoire, un appareil de pêche, le Zemi, un filet triangulaire monté sur une pirogue appelée Wam, dont la conception technique et l'application s'observent exclusivement, dans cette région fortement arrosée par des cours d'eau 
permanents. Ce travail s'attache à l'étude de l'instrument de capture, son installation et à la méthode de pêche des Kotoko, éléments fondamentaux de leur patrimoine culturel et technique halieutique, une tradition qui, aujourd'hui, a disparu des eaux du Logone et du Chari. C'est pour cette raison que les populations ont conçu des maquettes d'une échelle d'environ 1/50 qui sont exposées dans les musées ethnographiques de cette aire culturelle pour perpétuer la mémoire et la représentation matérielle de cet appareil de pêche à l'adresse de la jeune génération et des touristes de passage dans cette région. Ainsi, cette communication se propose de présenter le zemi, la technique et les moyens qu'il mobilise, son usage, son impact dans la socio-économique de l'aire culturelle kotoko, enfin de promouvoir son caractère patrimonial.

Economic activities determine the lifestyles of human groups and in some cases constitute the main pillars of civilization. The Kotokos along the Lake Chad are recognized as professional fishermen to a point where their history is linked to this activity. With time, they developed a fishing instrument called the Zemi; a triangular net mounted on a canoe called Wam, where its technical conception and application are exclusively observed in this region highly watered by the permanent streams. This work is focused on the Kotokos fish capturing instrument, its installation and method, a fundamental element of their cultural heritage and halieutic technique; a tradition which nowadays has disappeared from the Logone and Chari waters. It is for this reason that the population conceived a maquette of the scale 1:50, exhibited in ethnographic museums of this cultural area so as to perpetuate the memory and material representation of this fishing instrument for the younger generation and for tourists visiting this region. This paper proposes to present the zemi, the technique and the means required, its usage, its socio-economic impact of the Kotoko cultural area in order to promote its patrimonial identity.

\section{INDEX}

Mots-clés : histoire des techniques, kotoko, pêche, Afrique, savoirs traditionnels écologiques.

Keywords : history of technology, kotoko, fishing, Africa, traditional ecological knowledge (TEK) Index chronologique : Époque contemporaine Index géographique : Afrique

\section{AUTEUR}

\section{OUSMAN MAHAMAT ABBA}

Chargé de cours, Département des Beaux-Arts et Sciences du Patrimoine, Institut Supérieur du Sahel / Université de Maroua. 